ACCEPted In ApJ

Preprint typeset using IATEX style emulateapj v. 11/12/01

\title{
THERMAL INFRARED IMAGING AND ATMOSPHERIC MODELING OF VHS J125601.92-125723.9 b: EVIDENCE FOR MODERATELY THICK CLOUDS AND EQUILIBRIUM CARBON CHEMISTRY IN A HIERARCHICAL TRIPLE SYSTEM
}

\author{
Evan A. Rich ${ }^{1}$, Thayne Currie ${ }^{2}$, John P. Wisniewsim ${ }^{1}$, Jun Hashimoto ${ }^{3}$, Timothy D. \\ Brandt $^{4,5}$, Joseph C. Carson ${ }^{6}$, Masayuki Kuzuhara ${ }^{7}$, Taichi Uyama ${ }^{8}$ \\ Accepted in ApJ
}

\begin{abstract}
We present and analyze Subaru/IRCS $L^{\prime}$ and $M^{\prime}$ images of the nearby M dwarf VHS J125601.92125723.9 (VHS 1256), which was recently claimed to have a $\sim 11 \mathrm{M}_{J}$ companion (VHS $1256 \mathrm{~b}$ ) at $\sim 102$ au separation. Our adaptive optics images partially resolve the central star into a binary, whose components are nearly equal in brightness and separated by $00^{\prime \prime} 106 \pm 0$ "'001. VHS $1256 \mathrm{~b}$ occupies nearly the same near-infrared color-magnitude diagram position as HR 8799 bcde and has a comparable $L^{\prime}$ brightness. However, it has a substantially redder $H-M^{\prime}$ color, implying a relatively brighter $M^{\prime}$ flux density than for the HR 8799 planets and suggesting that non-equilibrium carbon chemistry may be less significant in VHS $1256 \mathrm{~b}$. We successfully match the entire SED (optical through thermal infrared) for VHS $1256 \mathrm{~b}$ to atmospheric models assuming chemical equilibrium, models which failed to reproduce HR $8799 \mathrm{~b}$ at $5 \mu \mathrm{m}$. Our modeling favors slightly thick clouds in the companion's atmosphere, although perhaps not quite as thick as those favored recently for HR 8799 bcde. Combined with the non-detection of lithium in the primary, we estimate that the system is at least older than 200 Myr and the masses of the stars comprising the central binary are at least $58 M_{J}$ each. Moreover, we find some of the properties of VHS 1256 are inconsistent with the recent suggestion that it is a member of the AB Dor moving group. Given the possible ranges in distance (12.7 pc vs. 17.1 pc), the lower mass limit for VHS $1256 \mathrm{~b}$ ranges from $10.5 M_{J}$ to $26.2 M_{J}$. Our detection limits rule out companions more massive than VHS $1256 \mathrm{~b}$ exterior to 6-8 au, placing significant limits on and providing some evidence against a second, more massive companion that may have scattered the wide-separation companion to its current location. VHS 1256 is most likely a very low mass (VLM) hierarchical triple system, and could be the third such system in which all components reside in the brown dwarf mass regime.
\end{abstract}

\section{INTRODUCTION}

Exoplanet surveys have recently measured the frequency of exoplanets as a function of a host of parameters like stellar mass, metallicity, orbital separation, and planetary mass (Winn \& Fabrycky 2015). These parameters help to inform our understanding of how and where exoplanets form. The observed frequency of gas giants at small $(<2 \mathrm{au}$ ) separations rises from $\sim 3 \%$ for $\mathrm{dM}$ stars to $\sim 14 \%$ for solar metallicity A-type stars; this dearth of massive planets around dM stars is consistent with theoretical predictions (Laughlin, Bodenheimer, \& Adams 2004) of the core accretion model (Pollack et al. 1996). At larger orbital separations (10s-100s of au), the frequency of gas giants around dM stars is $<6 \%$ (Bowler et al. 2015). Recent results from Kepler have shown both that the frequency of small mass planets at short orbital periods increases around low mass stars (Borucki et al. 2011; Howard et al. 2012) and there is a lack of planets larger than $2.5 R_{\oplus}$ surrounding dM stars at short orbital periods.

High contrast imaging investigations have similarly begun to discover gas giant exoplanets located at large or- bital separations from their stars (Fomalhaut b, Kalas et al. 2008, $\beta$ Pictoris b, Lagrange et al. 2009, 2010; HR 8799 bcde, Marois et al. 2010; $\kappa$ And. b, Carson et al. 2013; 51 Eri b ,Macintosh et al. 2015; HD 100546 bc ,Quanz et al. 2013, Currie et al. 2015a). While most of these directly imaged gas giants surround early-type stars, detections have been reported around Solar-analogs (GJ 504b, Kuzuhara et al. 2013) and dM stars (e.g. ROXs42B, Currie et al. 2014a; GU Psc, Naud et al. 2014). Yet the formation mechanisms responsible for these systems is still under debate. A growing number of objects with wide orbits and modest mass ratios (eg. HD 106906b, Bailey et al. 2014; ROXs42Bb, Currie et al. 2014a; 1RXSJ1609, Lafrenière et al. 2008; 2M J044144, Todorov et al. 2010) have led to suggestions that the planetary companion formed via a binary star-like process rather than the core accretion process (Low \& Lynden-Bell 1976, Bate 2009, Brandt et al. 2014).

Although binary stars are common (e.g. Raghavan et al. 2010), our understanding of the frequency of exoplanets around binaries and higher order systems remains limited.

${ }^{1}$ Homer L. Dodge Department of Physics, University of Oklahoma, 440 W. Brooks St., Norman, OK 73071, USA

2 National Astronomical Observatory of Japan, Subaru Telescope

3 Astrobiology Center, National Institutes of Natural Sciences, National Astronomical Observatory of Japan, 2-21-1 Osawa, Mitaka, Tokyo 181-8588 Japan

${ }^{4}$ Astrophysics Department, Institute for Advanced Study, Princeton, NJ, USA

5 NASA Sagan Fellow

6 Department of Physics and Astronomy, College of Charleston, 66 George Street., Charleston, SC 29424, USA

7 Department of Earth and Planetary Sciences, Tokyo Institute of Technology, 2-12-1 Ookayama, Meguro-ku, Tokyo 152-8551, Japan

8 Department of Astronomy, The University of Tokyo, 7-3-1, Hongo, Bunkyo-ku, Tokyo, 113-0033, Japan 
Since the discovery of the first exoplanet surrounding a binary host (Kepler-16b, Doyle et al. 2011), less than a dozen similar systems have been discovered by Kepler (Winn \& Fabrycky 2015 and references therein). The analysis of publicly available Kepler data led Armstrong et al. (2014) to conclude that the frequency of planets with $\mathrm{R}>6 \mathrm{R}_{\oplus}$ on periods of less than 300 days was similar to that of single star rates; however, this conclusion is critically dependent on the assumed planetary inclination distribution. While at least one bona fide planetary mass companion orbiting a binary has been imaged (ROXs $42 \mathrm{Bb}$ Simon et al. 1995; Ratzka, Kohler, \& Leinert 2005; Currie et al. 2014a) most dedicated direct imaging surveys for gas giant planets around binaries have not yielded any firm detections to-date (Thalmann et al. 2014).

Recently, Gauza et al. (2015) reported the detection of a planetary mass $\left(11.2_{-1.8}^{+9.7} \mathrm{M}_{J}\right)$ companion at a projected separation of $102 \pm 9$ au from its host star VHS J125601.92125723.9 (hereafter VHS 1256), described as a M7.5 object with an inferred mass from its bolometric luminosity of $73_{-15}^{+20} \mathrm{M}_{J}$, placing it near the hydrogen burning limit. The primary was estimated to have an age of 150-300 Myr from both kinematic membership in the Local Association and lithium abundance. At a distance of $12.7 \pm 1.0 p c$ measured from trigonometric parallax (Gauza et al. 2015), this made VHS 1256 the closest directly imaged planetary mass system to the Earth. Stone et al. (2016) reported a greater distance to VHS 1256 of $17.1 \pm 2.5$ pc based on spectrophotometry of the system.

From the standpoint of substellar atmospheres and atmospheric evolution, VHS $1256 \mathrm{~b}$ is a particularly unique object. Its near-infrared properties resemble those of the HR 8799 planets and a select few other young $(\mathrm{t} \lesssim 30 \mathrm{Myr})$ and very low mass $\left(\mathrm{M} \lesssim 15 M_{\mathrm{J}}\right)$ substellar objects, occupying roughly the same near-infrared color-magnitude space (Gauza et al. 2015; Faherty et al. 2016): a continuation of the L dwarf sequence to fainter magnitudes and cooler temperatures. Indeed, as shown by atmosphere modeling, the near-infrared properties of objects like HR 8799 bcde and 2M 1207 B reveal evidence for thicker clouds than field brown dwarfs of the same effective temperatures (Currie et al. 2011). VHS $1256 \mathrm{~b}$ then offers a probe of clouds at ages intermediate between these benchmark objects and Gyr-old field objects and thus some insights into the atmospheric evolution of low-mass substellar objects.

Furthermore, non-equilbrium carbon chemistry can be probed by new thermal infrared photometry, in particular at $M^{\prime}$ (e.g. Galicher et al. 2011) ${ }^{9}$. New thermal infrared data for VHS $1256 \mathrm{~b}$ allows us to assess the evidence for non-equilibrium chemistry for the objects at/near the deuterium burning limit and at ages older than HR 8799 bcde.

In this work, we present new adaptive optics imagery of VHS 1256, providing the first detections of its wideseparation companion in major thermal IR broadband filters, $L^{\prime}$ and $M^{\prime}$. We use these mid-infrared photometric points and optical and near-infrared photometry from Gauza et al. (2015) to perform the first atmospheric (for- ward) modeling of VHS $1256 \mathrm{~b}$ and the first assessment of how its thermal IR properties (e.g. carbon chemistry) compare to younger planet-mass objects with similar nearIR colors. Additionally, we report our independent determination of the primary's binarity, also reported in Stone et al. (2016), following our original work (Anthes Rich et al. 2015) with additional analyses. We will adopt the same nomenclature as Stone et al. 2016, referring to the close partially resolved binary as VHS $1256 \mathrm{~A}$ and B, and the wide companion as VHS $1256 \mathrm{~b}$.

After discussing our observations in Section 2, we search for new companions around VHS 1256 and investigate the binarity of VHS 1256 in Section 3. Next, we discuss improved photometry of VHS $1256 \mathrm{~A}, \mathrm{~B}$, and b at $L^{\prime}$ and $M^{\prime}$ in Section 4. Using the new $\mathrm{L}^{\prime}$ and $\mathrm{M}^{\prime}$ photometry, we assess the atmospheric properties of VHS $1256 \mathrm{~b}$ in Section 5. Finally, we discuss the implications of our study in Section 6 .

\section{OBSERVATIONS AND REDUCTIONS}

We observed VHS 1256 in $L^{\prime}\left(\lambda_{\text {center }}=3.77 \mu \mathrm{m}\right)$ and $M^{\prime}\left(\lambda_{\text {center }}=4.68 \mu \mathrm{m}\right)$ bands on 2015 June 6 using the Infrared Camera and Spectrograph (IRCS; Kobayashi et al. (2000)) on the $8.2 \mathrm{~m}$ Subaru Telescope, along with the Subaru AO-188 adaptive optics (AO) system (Hayano et al. 2008, 2010) in laser guide star (LGS) mode. All observations were made with IRCS having a plate scale of 20.57 mas pix $^{-1}$. For the L' band, we utilized a 0.1 second exposure time along with 100 co-adds to achieve 10 second exposures per dither frame. We obtained a total of 13 dither sets, using a 5 point dither per set, yielding a total on-source integration time of 650 seconds. For our $M^{\prime}$-band imaging, we utilized a 0.06 second exposure time along with 100 co-adds to achieve 6 second exposures per dither frame. We observed VHS 1256 for a total of 3 dither sets, using a 5 point dither per set, yielding a total on-source integration time of 90 seconds. All data were obtained in angular differential imaging (ADI) mode; a total field rotation of $17^{\circ} .4$ and $2^{\circ} .8$ was achieved in $L^{\prime}$ and $M^{\prime}$ respectively.

We utilized two approaches to detect VHS 1256 b. First, we simply derotated each image to true north after sky subtraction. We inspected the quality of each image set, and determined that the AO performance was subpar during $7 L^{\prime}$ frames (70 seconds of integration); hence, we removed these data before median combining frames. Using an identical process, we reduced the $M^{\prime}$ data and removed 6 of the 15 frames (54 seconds of integration) due to subpar AO performance.

Second, we utilized advanced PSF subtraction methods and a different approach with image combination to compensate for the shorter integration time and brighter sky background in $M^{\prime}$ and obtained slightly deeper limits in $L^{\prime}$. We use A-LOCI as in Currie et al. $(2012,2015 \mathrm{~b})$ with a large optimization area (500 PSF footprints) and rotation gap (1.5 times the diffraction limit at $\mathrm{L}^{\prime}$ and $\mathrm{M}^{\prime}$ ) combined with a moving pixel mask to flatten the background with-

9 The existing W2 photometry reported in Gauza et al. (2015) covers a far wider bandpass ( $4-5 \mu m)$. Much of this wavelength range is far less sensitive to carbon monoxide opacity at relevant temperatures that is a tracer of non-equilbrium carbon chemistry, while $M^{\prime}$ is far more (uniquely) sensitive (e.g. see Figure 7 in Currie et al. 2014b).

10 as shown by Galicher et al. (2011), advanced methods usually used for point-spread function (PSF) subtraction can in some cases better flatten the sky background, especially in $\mathrm{M}^{\prime}$. As VHS $1256 \mathrm{~b}$ is located about $8^{\prime \prime}$ from the primary, even a small $2^{\circ} .8$ parallactic motion is sufficient to apply PSF subtraction methods like LOCI (Lafreniere et al. 2007) 
out removing signal from VHS $1256 \mathrm{~b}^{10}$. We then applied a $4 \lambda / \mathrm{D}$ spatial filter and combined the derotated data to obtain a mean image using a $3-\sigma$ outlier rejection. This allowed us to obtain a higher signal-to-noise detection of VHS $1256 \mathrm{~b}$, which was barely detectable in $M^{\prime}$ at the 3- $\sigma$ level using the first image processing method, obtaining about a $40 \%$ deeper background limit at $L^{\prime}$.

We utilized observations of the $L^{\prime}$ standard FS 138 (van der Bliek, Manfroid, \& Bouchet 1996) obtained on 2015 June 7 using NGS and observations of the $M^{\prime}$ standard HR 5384 (van der Bliek, Manfroid, \& Bouchet 1996) obtained on 2015 June 8 without AO to transform our photometry to a standard system. We did not obtain successful images of a dense stellar cluster that would be needed to construct a robust distortion correction for our $L^{\prime}$ and $M^{\prime}$ imagery; hence, we have included no such correction to our data. Our lack of a distortion correction introduces some uncertainty into VHS 1256 b's separation, but does not affect our other results.

\section{SEARCH FOR ADDITIONAL COMPANIONS}

\subsection{Point Source Detections and Detection Limits in the $L^{\prime}$ and $M^{\prime}$ Field of View}

Our fully reduced $L^{\prime}$ - and $M^{\prime}$-band imagery (Figure 1) clearly reveals the presence of both the composite source of VHS $1256 \mathrm{~A}$ and B (A+B) and VHS $1256 \mathrm{~b}$ reported by Gauza et al. (2015). We detect the companion at SNR $\sim 130$ (100) in the $L^{\prime}$ data and at 4.5 (3) in the $M^{\prime}$ data using the A-LOCI (classical) reduction, where the latter detection is roughly comparable to the detection significance of HR 8799 bcd in Galicher et al. (2011). We do not identify any additional point sources.

We determined the centroid positions of both objects, and found that VHS $1256 \mathrm{~b}$ is located $8{ }^{\prime \prime} 13 \pm 0^{\prime \prime} 04$ from VHS $1256 \mathrm{~A}+\mathrm{B}$ at a position angle of $217.8^{\circ} \pm 0.3^{\circ}$ in $L^{\prime}$ and $8^{\prime \prime} .17 \pm 0 . \prime 04$ at a position angle of $217.8^{\circ} \pm 0.3^{\circ}$ in $M^{\prime}$ (Table 1). This separation is consistent with the angular separation observed by Gauza et al. (2015) of 8."06 $\pm 00^{\prime \prime} 03$ at a position angle of $218.1^{\circ} \pm 0.2^{\circ}$. Note that we did not utilize a distortion correction.

To compute the 5- $\sigma$ point source detection limit in $L^{\prime}$, we followed standard methods used in high-contrast imaging data (e.g. Currie et al. 2015b). We convolved the image with a gaussian profile having a FWHM set by the observed FWHM of VHS $1256 \mathrm{~b}$ and determination the robust standard deviation of convolved pixels at each angular separation. The limiting 5- $\sigma$ point source detection limits of our $L^{\prime}$ imagery based on this method is roughly 16.4 mag exterior to 2.5 ( $\mathrm{r}_{\text {proj }} \sim 32$ au for $d=12.7 p c$ ), degrading to VHS 1256 b's brightness at $0{ }^{\prime \prime} 5$. Due to the thermal background, limited integration time, and the partial FOV coverage, the $M^{\prime}$ imagery do not provide stringent constraints on the presence of additional point sources compared to the $L^{\prime}$ imagery.

\subsection{Analysis of the Primary: Evidence of Multiplicity}

It is apparent from visual inspection of Figure 1 that the VHS $1256 \mathrm{~A}+\mathrm{B}$ is much more elongated in both $L^{\prime}$ and $M^{\prime}$-bands than VHS $1256 \mathrm{~b}$. In fact, simple gaussian fits to the data reveal that the FWHM of the central star is

\footnotetext{
11 http://nicky.vanforeest.com/misc/fitEllipse/fitEllipse.html
}

significantly broader than the companion in both bandpasses. Zooming in on the region around VHS $1256 \mathrm{~A}+\mathrm{B}$, Figure 2 demonstrates that the central star is comprised of two marginally resolved sources. Using a modified elliptical least squares minimization fitting routine written by Nicky van Foreest ${ }^{11}$, we compute the average ellipticity $\left.\left(\mathrm{e}=\left(\left(\mathrm{a}^{2}-\mathrm{b}^{2}\right) / \mathrm{a}^{2}\right)^{0.5}\right)\right)$ of our sources. The average ellipticity of the central star, $0.85 \pm 0.02$ and $0.825 \pm 0.005$ in the $L^{\prime}$ and $M^{\prime}$ bands respectively, is significantly greater than the average ellipticity observed for the companion $\left(0.48 \pm 0.03\right.$ in $\left.L^{\prime}\right)$. These results all indicate that the central star is clearly comprised of the superposition of two separate sources.

To help ascertain the probability that the observed multiplicity of VHS $1256 \mathrm{~A}$ and B arises from chance alignment of VHS 1256 with a background source, we utilize archival WISE W1-band imagery, which has a similar band-pass as our $L^{\prime}$-band imagery. The local density of W1 sources between 10.2 and $10.8 \mathrm{mag}$ is $\sim 34 \mathrm{deg}^{-2}$. Hence, the chance alignment of two sources, within 0 "' 106 is $5 \times 10^{-6 \%}$.

\section{PHOTOMETRY}

Aperture photometry was performed on all components of the VHS 1256 system. Both the L' photometry of VHS $1256 \mathrm{~A}+\mathrm{B}(9.757 \pm 0.04$ magnitude) and VHS $1256 \mathrm{~b}$ (12.93 \pm 0.02 magnitude) and the $\mathrm{M}^{\prime}$ photometry of VHS $1256 \mathrm{~A}+\mathrm{B}(9.65 \pm 0.04$ magnitude) and VHS $1256 \mathrm{~b}$ (12.66 \pm 0.26 magnitude) are broadly consistent with the literature (Gauza et al. 2015) WISE W1 and W2 photometry (Table 2). We utilized a 30 pixels radius for aperture photometry. In addition to the standard uncertainty terms (poisson noise and standard deviation of background), we included read noise, shot noise, and the errors from the zero point terms. We do not include error terms due to variations in the atmospheric transmission during our observations, or due to variations that arise between the use of NGS versus LGS observing modes. Note that our $L^{\prime}$ and $M^{\prime}$ VHS $1256 \mathrm{~b}$ photometry improves on the respective WISE W1 and W2 errors previously presented.

The relative astrometry and flux of VHS $1256 \mathrm{~A}$ and $\mathrm{B}$ were constrained by fitting a linear combination of two PSFs to our $L^{\prime}$ images; we adopt VHS $1256 \mathrm{~b}$ as the PSF. We first align the dithered images of the central binary by maximizing their cross-correlation and then combine them by averaging. Averaging the frames is optimal in the case of Gaussian errors; in our case, it reduces the noise by nearly a factor of 2 compared with a median combination.

We use bicubic spline interpolation to translate the PSF of VHS $1256 \mathrm{~b}$. We then simultaneously adjust the positions and normalizations of two PSFs to minimize

$$
\chi^{2}=\sum \frac{\left(\mathrm{PSF}_{1}+\mathrm{PSF}_{2}-\text { data }^{2}\right.}{\sigma_{\mathrm{ph}}^{2}+\sigma_{\mathrm{bg}}^{2}}
$$

where $\sigma_{\mathrm{ph}}^{2}$ is the variance from photon noise and $\sigma_{\mathrm{bg}}^{2}$ is the variance from read noise and the thermal background. We estimate $\sigma_{\mathrm{ph}}^{2}$ from the instrument gain and measure $\sigma_{\mathrm{bg}}^{2}$ from empty regions of the image. The variance is the sum of variance in the image and scaled variance from the noisy companion PSF. In practice, most of the noise arises from scaling the companion PSF, as the noise increases linearly with the scaling factors. 
A good fit should have $\chi^{2} \approx N_{\text {pix }}$, where $N_{\text {pix }}$ is the number of pixels in the region being fit. With a simple estimate of the background and photon noise, we find that our best-fit $\chi^{2}$ is about four times the number of pixels; we therefore increase our estimated uncertainties to achieve a reduced $\chi^{2}$ of unity. After rescaling our noise to achieve a minimum $\chi^{2}=N_{\text {pix }}$, we compute our confidence regions by integrating the likelihood

$$
\mathcal{L}=\exp \left[-\chi^{2} / 2\right]
$$

and computing the regions containing $68 \%$ of the likelihood (for our $1 \sigma$ confidence intervals).

Figure 3 shows the results of this fit: the observed $L^{\prime}$ intensity of VHS $1256 \mathrm{~A}$ and B (left panel), the bestfit translated and scaled linear combination of companion PSFs (middle panel), and the residuals (right panel), expressed in units of the peak intensity of the original data. The fit is good to a few percent near the PSF cores, comparable to the expected uncertainties from the noise and from interpolations. Spatial correlations in the noise are visible in the residual image even far from the PSF core; it is these correlations that force us to scale our errors. A full treatment of the problem would modify $\chi^{2}$ (Equation 1) to account for the data's non-diagonal covariance matrix. Table 1 lists our fitted parameters: a separation of $0{ }^{\prime \prime} 106 \pm 0 . \prime 001$ (5.18 \pm 0.05 pixels), a position angle of $-7^{\circ} .6 \pm 0.5$, and a flux ratio of $1.03 \pm 0.01$. We thus obtain $1 \%$ measurements of the relative photometry and separation.

\section{ATMOSPHERIC PROPERTIES OF VHS 1256 B}

The discovery work by Gauza et al. (2015) suggested that VHS $1256 \mathrm{~b}$, based on its spectral shape and colormagnitude diagram position, is a red $\mathrm{L} / \mathrm{T}$ transition object similar to HR 8799 bcde (e.g. Currie et al. 2011; Bonnefoy et al. 2016). With SED information spanning the red optical to mid-infrared, we can more thoroughly compare VHS 1256 b's properties to those of other substellar objects and fit atmosphere models with a range of assumptions about clouds to reproduce VHS1256 b's spectrum. Many free floating $\mathrm{L} / \mathrm{T}$ dwarfs with red optical to near-infrared photometry and spectra have been studied (e.g. Knapp et al. 2004). However, even considering young brown dwarfs as well as the field, VHS 1256 b occupies an extremely sparsely populated near-infrared color-magnitude diagram position shared by planet mass objects like HR 8799 bcde and 2M 1207 B (Faherty et al. 2016). Furthermore, these objects are young, typically less than $\sim 30 \mathrm{Myr}$ old (Faherty et al. 2016), while VHS 1256 is at least older than 200 Myr (Stone et al. 2016, sect 6.2 of this work).

While the similarity of VHS 1256 b's near-infrared colors to those of HR 8799 bcde suggest that their atmospheric properties likewise share some similarity (e.g. perhaps thick clouds), the addition of thermal infrared data in this work allows us to further quantify this feature and assess whether or not VHS $1256 \mathrm{~b}$ shows clear evidence for non-equilibrium carbon chemistry. Compared to HR 8799 bcde, VHS 1256 b's available suite of photometry extends far bluer, into the optical. Thus, the companion provides a new test of atmospheric models used to reproduce at least some bona fide directly-imaged planets. Furthermore, as shown in Faherty et al. (2016), young L dwarfs tend to follow a reddened version of the field sequence. Comparing VHS $1256 \mathrm{~A}+\mathrm{B}$ 's colors to those of the field sequence and young associations like AB Dor can then provide a very coarse assessment of the system's youth.

\subsection{Mid-Infrared Colors of VHS 1256 A, B, and $b$}

Figure 4 compares VHS 1256 A, B, and b's near-infrared and mid-infrared color magnitude diagrams (CMDs) to the field sequence, planetary companions like HR 8799 bcde, and substellar objects in $\mathrm{AB}$ Dor $(t \sim 125 \mathrm{Myr})$. The primary components to VHS 1256 appear indistinguishable from the field sequence and bluer than AB Dor members. Even if VHS $1256 \mathrm{~b}$ is at $17.1 p c$ and thus intrinsically brighter, it still appears somewhat "under luminous", along an extension of the L dwarf sequence to fainter magnitudes and presumably lower effective temperatures. Depending on the distance, VHS 1256 b's $L^{\prime} / \mathrm{H}-L^{\prime}$ position is either consistent with HR 8799 b's or appears more comparable to the inner three planets.

In contrast, VHS $1256 \mathrm{~b}$ is significantly brighter at $M^{\prime}$, relative to HR 8799 bcde. VHS 1256 b's H- $M^{\prime}$ color is $0.6-$ 1.1 magnitudes redder that HR $8799 \mathrm{bcd}$, suggesting that at $M^{\prime}$ it is roughly 1.7-2.7 times as bright. The Leggett et al. (2010) and Dupuy \& Liu (2012) compilations of M/L/T objects (Figure 4) are more sparsely populated in $M^{\prime}$ for late $\mathrm{L}$ and $\mathrm{T}$ dwarfs making comparisons with the field sequence difficult. As the faint $M^{\prime}$ brightnesses for $\mathrm{L} / \mathrm{T}$ transition objects and the HR 8799 planets signaled evidence for non-equilibrium carbon chemistry (Galicher et al. 2011), VHS 1256 b's brighter $M^{\prime}$ flux density may indicate that non-equilibrium carbon chemistry is not significant for every $\mathrm{L} / \mathrm{T}$ transition object.

\subsection{Atmospheric Modeling: Methodology}

We perform atmospheric forward modeling to estimate VHS 1256 b's temperature, surface gravity, and radius, and to see if its photometry can be reproduced by atmospheric models in chemical equilibrium. We follow the studies of Currie et al. (2011) and Madhusudhan et al. (2011) for HR 8799's planets and Burrows et al. (2006) for field brown dwarfs, comparing the data with atmosphere models from A. Burrows covering a range of parameterized cloud prescriptions but all assuming chemical equilibrium.

We consider the "E60" models, which, have a modal particle size of $60 \mu \mathrm{m}$ and clouds sharply truncated at depth, well below the planet photosphere. We then consider "A60" and "AE60" cloud models, which have an identical modal particle size but simulate "very thick" and "thick" cloud model presciptions (see Burrows et al. 2006 and Madhusudhan et al. (2011) for details). From the model fitting, we identify those consistent with the data to within 3- $\sigma$ using a simple $\chi^{2}$ threshold, setting the minimum photometry uncertainty to be $10 \%$ as in Currie et al. (2011). This method then yields a plausible range of temperature, surface gravity, radius, luminosity and mass from the ensemble of acceptably-fitting models.

We perform two sets of model fits, one where we allow the planet radius to freely vary and another when we pin it to values implied in the Burrows et al. (2001) evolutionary models. We assess how the system's uncertain distance affects our best-fit derived by performing fits, for 
the fixed-radius case, assuming either distances (12.7 and $17.1 p c)$.

\subsection{Atmospheric Modeling: Results}

Figure 5 displays best-fit models using each cloud model assumption and Tables 3 and 4 summarize our model fitting results. VHS 1256 b's SED cannot be reproduced by the thin-cloud, E60 models: like HR 8799's planets, such models badly under-predict the companion's brightness at the shortest wavelengths and over-predict it in the thermal infrared (see Currie et al. 2011). The A60, very-thick cloud models yield a drastically improved fit at temperatures between $900 K$ and $1200 K$, where a model with $T_{\text {eff }}$ $=1000 \mathrm{~K}, \log (\mathrm{g})=4.5$ is marginally consistent with the optical to mid-infrared SED at the 3- $\sigma$ confidence limit.

Models with slightly thinner clouds, AE60, fare better, yielding a wider range of temperature and surface gravity phase space able to match the data. The best-fit values systematically skew towards lower temperatures and surface gravities, where the best fit model has $T_{\text {eff }}=800 \mathrm{~K}$ and $\log (\mathrm{g})=3.8$ when the radius can freely vary and has a slightly higher gravity $(\log (\mathrm{g})=4.1)$ when the radius is fixed.

Assuming a distance of $17.1 p c$, the implied luminosity of VHS 1256 b ranges between $\log \left(\mathrm{L} / \mathrm{L}_{\odot}\right)=-4.79$ to -4.95 . Adopting the $12.7 p c$ distance yields a luminosity consistent with that previously estimated by Gauza et al. (2015): $\log \left(\mathrm{L} / \mathrm{L}_{\odot}\right)=-5.06$ to -5.24 . We will estimate the mass of VHS $1256 \mathrm{~b}$ by using these luminosities in section 6.2. While successful at reproducing VHS 1256 b's SED, the derived model fit parameters could be revised by future modeling efforts. For instance, the implied radii for best-fit models are generally larger than predicted for substellar object's with ages greater than $150 \mathrm{Myr}$ (see Baraffe et al. 2003), and thus similarly the temperatures could be slightly larger than implied by our analysis.

\section{DISCUSSION}

\subsection{Binarity of the Central Source}

Subaru/IRCS AO $L^{\prime}$ and $M^{\prime}$ imagery has clearly revealed that the central source of the VHS 1256 system is comprised of two objects (Figure 1) that have similar relative brightness $\left(L^{\prime}=10.5\right.$ and 10.54 magnitude respectively). Such binarity is observed in $22_{-4}^{+6} \%$ of very low mass stars (Duchene \& Kraus 2013). Gauza et al. (2015) assigned the central source a spectral classification of M7.5, based on optical (M7.0) and IR (M8.0) spectral classifications. We speculate that the minor differences in the optical versus IR spectral classifications derived by Gauza et al. (2015) could be caused by minor differences in the spectral classifications of the binary components. At the observed distance to VHS $1256(12.7 \pm 1.0$ pc; Gauza et al. 2015), the 0 ". $103 \pm 0$.'001 projected separation between the binary components corresponds to a projected physical separation of $\sim 1.3$ au. Our results on the binarity of the central source are consistent with those independently and recently reported by Stone et al. (2016).

\subsection{System Age and Component Masses}

Gauza et al. (2015) suggested a system age of 150-300 $\mathrm{Myr}$, based on the lack of observed $\mathrm{Li}$ in the system and kinematic age constraints from being a Local Association member. However, with the discovery that the central source is a binary (Stone et al. 2016) and our independent verification of VHS $1256 \mathrm{~A}$ and B in the $L^{\prime}$-band, we can reassess the age limits of the system. Using the nominal distance $(12.7 \mathrm{pc})$ and the absolute magnitude $\left(\mathrm{M}_{L^{\prime}} ; 10.0 \pm 0.2\right)$, the 300 Myr upper limit age suggested by Gauza et al. (2015) results in an inferred mass for VHS $1256 \mathrm{~A}$ or $\mathrm{B}$ of $47 \mathrm{M}_{J}$. However such a mass would be too small to destroy Li (Allard 2014) and produce the nondetection of this line (Figure 6). Rather, at this adopted distance the lower limit age of VHS 1256 must be $>400$ Myr to produce VHS $1256 \mathrm{~A}$ and B with our observed $\mathrm{M}_{L^{\prime}}$ and the lack of $\mathrm{Li}$ in the system's spectra. If one assumes the new distance of 17.1 pc proposed by Stone et al. (2016) and the corresponding absolute magnitude of the central components $\left(\mathrm{M}_{L^{\prime}} ; \sim 9.4 \pm 0.3\right)$, the lower age limit is $>$ 200 Myrs (Figure 5). This is broadly consistent with the lower age limit proposed by Stone et al. (2016) of $280_{-50}^{+40}$. Note we used models from Allard (2014), while Stone et al. used models from Chabrier et al. (2000).

Stone et al. (2016) suggested that VHS 1256 was consistent with being a member of the AB Dor moving group, based on analysis of its UVW kinematics and a $66.85 \%$ membership probability predicted by the BANYAN II software tool (Malo et al. 2013; Gagne et al. 2014). Our own investigation suggests that it still has a $28 \%$ chance of being in the "young field" (age up to 1 Gyr). Additionally, VHS $1256 \mathrm{~b}$ is a clear outlier in UWV space $(\sim 8$ $\pm 1.7 \mathrm{~km} \mathrm{~s}^{-1}$ from the core of AB Dor. (J. Gagne, pvt. comm.). Furthermore, membership in the $149_{-19}^{+51}$ Myr AB Dor moving group (Bell et al. 2015) is inconsistent with the lower age limit of $280_{-50}^{+40}$ proposed by Stone et al. (2016) and marginally inconsistent with our lower limit of 200-400 Myr. Moreover, the near-to-mid infrared colors of VHS 1256 A appear indistinguishable from those in the field and potentially bluer than AB Dor members (Figure 4. Thus, it is not clear that VHS 1256 is a member of the AB Dor moving group, as suggested by Stone et al. (2016).

As shown in Figure 6, the minimum mass of each central component of VHS 1256 (A and B) is $>58 \mathrm{M}_{J}$ for both of the distances discussed above. This implies that the wide companion, VHS $1256 \mathrm{~b}$, has a minimum mass ranging from 10.5 to $26.2 \mathrm{M}_{J}$, as shown in Table 5. The large range is due to the uncertainty in the distance (12.7 or $17.1 \mathrm{pc}$ ) and the range in bolometric luminosities from atmospheric fitting (section 5). Though the lower estimate does dip below the deuterium burning limit, the companion is most likely in the brown dwarf regime.

\subsection{Additional Companions and Formation}

We detected no other point source companions in our field of view $(\mathrm{FOV}), \sim 16^{\prime \prime} .5 \mathrm{x} \sim 16^{\prime \prime} .5$ in $L^{\prime}$ and $\sim 9^{\prime \prime} .3 \mathrm{x} \sim 9^{\prime \prime} .3$ in $M^{\prime}$, down to our 5 - $\sigma$ sensitivity limits shown in Figure 7. of $13.2\left(L^{\prime} ; 12.5 \mathrm{mag}\right.$ at $\left.17.1 \mathrm{pc}\right)$. Assuming a distance of 12.7 (17.1) $p c$, minimum system age of $>400$ (200) Myr, and no flux reversal at $L^{\prime}$ (i.e. that more massive objects are fainter), we can therefore exclude the presence of additional companions more massive than VHS $1256 \mathrm{~b}$ beyond $6(8)$ au. For most of the semi major axis space we probe, comparisons with Baraffe et al. (2003) imply that companions down to $3-5 M_{\mathrm{J}}$ can be excluded if the system 
is 200-400 Myr old.

Because we have failed to identify other substellar companions orbiting the primary, this severely restricts the possibility that VHS $1256 \mathrm{~b}$ was scattered to its present orbit by dynamical interactions with another, unseen planet. Thus far, searches for close-in substellar companions to stars with imaged (near) planet-mass companions at 100500 au have failed to identify potential scatterers, suggesting that this class of objects formed in situ either from protostellar disk or molecular cloud fragmentation (Bryan et al. 2016).

Furthremore, the mass ratio (q) of VHS $1256 \mathrm{~b}$ (M 18.4 $\mathrm{M}_{J}$; median lower limit between 10.2-26.2 $\mathrm{M}_{J}$ ) to VHS $1256 \mathrm{~A}+\mathrm{B}\left(\mathrm{M} \geq 116 M_{J}\right)$ is $\sim 0.16$. This mass ratio is substantially larger than that observed for other imaged planetary systems such as HR $8799\left(\mathrm{q} \sim 5 * 10^{-3}\right.$; Fabrycky \& Murray-Clay 2010) and ROXs 42B (q $\sim 0.008-0.01$; Currie et al. 2014a). Rather, it is more similar to that observed for low mass BDs $(\mathrm{q} \sim 0.01-0.9$; eg. see Figure 4, Currie et al. 2014a and citations there in). We suggest this is indicative that the system formed via some form of fragmentation, i.e. a binary-star-like formation mechanism, rather than core accretion (Pollack et al. 1996). Stone et al. 2016 reached a similar conclusion of the binary-starlike formation.

\subsection{Atmospheric Modeling}

Although VHS 1256 b occupies a similar near-IR colormagnitude space to HR 8799 bcde (Gauza et al. 2015), its significantly older age than the HR 8799 system enables one to probe a different time frame in planet/brown dwarf atmospheric evolution. VHS $1256 \mathrm{~b}$ and HR $8799 \mathrm{bcd}(\mathrm{e}$ ?) have different spectral energy distributions at the longest wavelengths probe $\left(M^{\prime} / 4.7 \mu m\right)$. In the now-standard picture of understanding the atmospheres of the youngest and lowest-mass L/T objects, thick clouds and non-equilibrium carbon chemistry both are due to the objects' low surface gravity (e.g. Marley et al. 2012). That VHS 1256 b thus far lacks evidence for non-equilibrium carbon chemistry may complicate this picture, suggesting some decoupling of gravity's two effects or that VHS 1256 b's gravity is high enough that non-equilibrium effects are less obvious than they are for, say, HR 8799 b.

Higher signal-to-noise detections in $M^{\prime}$ and photometry in the 3-4 $\mu \mathrm{m}$ range probing methane will allow us to better clarify VHS 1256 b's carbon chemistry. Multiple lines in $J$ band resolvable at medium resolution could better clarify the companion's surface gravity. With other, similar objects detected at a range of ages, we can better map out the atmospheric evolution of objects of a given mass as well as the diversity of objects occupying the same reddened L/T transition region where VHS $1256 \mathrm{~b}$ and bona fide planets like HR 8799 bcde reside.

\subsection{System Architecture}

The VHS 1256 hierarchical triple system is poised to become an important contributor to our understanding of VLM systems. It represents the third hierarchical triple system comprised solely of brown dwarf-mass components known (Bouy et al. 2005; Radigan et al. 2013). Given this projected separation (1.3 au) and associated approximate orbital period ( $\sim 4.7$ years) of the central binary in VHS 1256 , future AO spectroscopic monitoring of the system is poised to determine dynamical masses of all components of the triple system, which should help constrain evolutionary models (see e.g. Dupuy et al. 2010). Since at least some brown dwarf binaries are believed to form via the disintegration of triple systems, and the third body in such systems are most likely also brown dwarf mass object (Reipurth \& Mikkola 2015), robustly determining the fundamental properties of the few known triple systems like VHS 1256 could help test the predictions of dynamical simulations of BD formation and evolution.

We thank Sarah Schmidt for thoughtful discussions about L/T dwarfs and multiplicity and Jonathan Gagne for helpful comments on VHS 1256's possible membership in different moving groups. We acknowledge support from NSF-AST 1009314 and NASA's Origins of Solar Systems program under NNX13AK17G. This work was performed in part under contract with the Jet Propulsion Laboratory (JPL) funded by NASA through the Sagan Fellowship Program executed by the NASA Exoplanet Science Institute. This work is also based on data collected at Subaru Telescope, which is operated by the National Astronomical Observatory of Japan. The authors recognize and acknowledge the significant cultural role and reverence that the summit of Mauna Kea has always had within the indigenous Hawaiian community. We are most fortunate to have the opportunity to conduct observations from this mountain.

\section{REFERENCES}

Allard, F. 2014 in Exploring the Formation and Evolution of Planetary Systems, IAU Proc. 299, 271

Anthes Rich, E., Wisniewski, J. P., Hashimoto, J., et al. 2015, AAS/Division for Extreme Solar Systems Abstracts, 3, 117.10

Armstrong, D.J., Osborn, H.P., Brown, D.J.A., Faedi, F., Gomez Maqueo Chew, Y., Martin, D.V., Pollacco, D., \& Udry, S. 2014, MNRAS, 444, 1873

Bailey, V., Meshket, T., Reiter, M. et al. 2014, ApJL, 780, 4

Baraffe, I., Chabrier, G., Barman, T. S., et al., 2003, A\&A, 402, 701

Bate, M. R. 2009, MNRAS, 392, 590

Bell, C.P.M., Mamajek, E.E., \& Naylor, T. 2015, MNRAS, 454, 593

Borucki, W.J., Koch, D.G., Basri, G. et al. 2011, ApJ, 736, 19

Bonnefoy, M., Zurlo, A., Baudino, J.-L., et al., 2016, A\&A, in press

Bouy, H., Martin, E.L., Brandner, W., \& Bouvier, J. 2005, AJ, 129, 511

Bowler, B. P., Liu, M. C., Shkolnik, E. L., \& Tamura, M. 2015, ApJS, 216,7
Brandt, T.D., Kuzuhara, M., McElwain, M.W. et al. 2014, ApJ, 786, 1

Bryan, M., et al., 2016, ApJ, in press

Burrows, A., Hubbard, W. B., Lunine, J. I., Liebert, J., 2001, Review of Modern Physics, 73, 719

Burrows, A., Sudarsky, D., Hubeny, I., 2006, ApJ, 640, 1063

Chabrier, G., Baraffe, I., Allard, F., \& Hauschildt, P. 2000, ApJ, 542, 464

Carson, J., Thalmann, C., Janson, M., et al. 2013, ApJ, 763, L32

Currie, T., Burrows, A., Itoh, Y., et al., 2011, ApJ, 729, 128

Currie, T., Debes, J. H., Rodigas, T. J., et al., 2012, ApJ, 760, L32

Currie, T., Daemgen, S., Debes, J., Lafreniere, D., Itoh, Y., Jayawardhana, R., Ratzka, T., \& Correia, S. 2014, ApJL, 780, 30

Currie, T., Burrows, A., Girard, J. H., et al. 2014, ApJ, 795, 133

Currie, T., Cloutier, R., Grady, C., et al., 2015, ApJ, 814, L27

Currie, T., Lisse, C., Kuchner, M., et al., 2015, ApJ, 807, L7

Duchene, G. \& Kraus, A. 2013, ARA\&A, 51, 269 
Doyle, L.R., Carter, J.A., Fabrycky, D.C. et al. 2011, Science, 333, 1602

Dupuy, T. J., Liu, M. C., Bowler, B. P., et al. 2010, ApJ, 721, 1725

Dupuy, T. J., \& Liu, M. C. 2012, ApJS, 201, 19

Hayano, Y., Takami, H., Guyon, O. et al. 2008, in Proc. SPIE, Adaptive Optics Systems, Eds. N. Hubin, Claire Max, \& P. Wizinowich, Vol 7015, 10

Hayano, Y., Takami, H., Oya, S. et al. 2010, in Proc. SPIE, Vol. 7736,0

Howard, A.W., Marcy, G.W., Bryson, S.T. et al. 2012, ApJS, 201, 15

Fabrycky, D. C., \& Murray-Clay, R. A. 2010, ApJ, 710, 1408

Faherty, J. K., Riedel, A. R., Cruz, K. L., et al. 2016, arXiv: 1605.07927

Gagne, J., Lafreniere, D., Doyon, r., Malo, L., \& Artigau, E. 2014, ApJ, 783, 121

Gauza, B., Bejar, V.J.S., Perez-Garrido, A. et al. 2015, ApJ, 804, 96

Galicher, R., Marois, C., Macintosh, B., et al., 2011, ApJ, 739, L41

Hinz, P., Rodigas, T.J., Kenworthy, M.A. et al. 2010, ApJ, 716, 417

Kalas, P.G., Graham, J.R., Chiang, E. et al. 2008, Science, 322, 1345

Knapp, G. R., Leggett, S. K., Fan, X., et al. 2004, AJ, 127, 3553

Kobayashi, H., Kawaguchi, N., Fujisawa, K. et al. 2000, in Proc. SPIE, Optical and IR Telescope Instrumentation and Detectors, eds M. Iye \& A. F. Moorwood, Vol. 4008, 1056

Kuzuhara, M., Tamura, M., Kudo. T. et al. 2013, ApJ, 774, 11

Lafreniere, D., Marois, C., Duyon, R., et al. 2007, ApJ, 660, 770

Lafrenière, D., Jayawardhana, R., \& van Kerkwijk, M. H. 2008, ApJ, 689, L153

Lagrange, A.-M., Gratadour, D., Chauvin, G., et al. 2009, A\&A, 493, L21

Lagrange, A.-M., Bonnefoy, M., Chauvin, G., Apai, D., Ehrenreich, D., Boccaletti, A., Gratadour, D., Rouan, D., Mouillet, D., Lacour, S., \& Kasper, M. 2010, Science, 329, 57

Laughlin, G., Bodenheimer, P., \& Adams, F.C. 2004, ApJL, 612, 73
Leggett, S. K., Golimowski, D. A., Fan, X., et al. 2002, ApJ, 564, 452

Leggett, S. K., Burningham, B., Saumon, D., et al., 2010, ApJ, 710, 1627

Liu, M.C., Magnier, E. A¿, Neacon, N. R., et al., 2013, ApJ, 777, L20

Low, C., \& Lynden-Bell, D. 1976, MNRAS, 176, 367

Macintosh, B., Graham, J.R., Barman, T. et al. 2015, Science, 350, 64

Madhusudhan, N., Burrows, A., Currie, T., 2011, ApJ, 737, 34

Malo, L., Doyon, R., Lafreniere, D., et al. 2013, ApJ, 762, 88

Marley, M., Saumon, D., Cushing, M., et al., 2012, ApJ, 754, 135

Marois, C., Zuckerman, B., Konopacky, Q.M., Macintosh, B., \& Barman, T. 2010, Nature, 468, 1080

Naud, M.-E., Artigau, E., Malo, L. et al. 2014, ApJ, 787, 5

Quanz, S., Meyer, M. R., Kenworthy, M., et al., 2013, ApJ, 766, L1

Pollack, J. B., Hubickyj, O., Bodenheimer, P., et al. 1996, Icarus, 124,62

Radigan, J., Jayawardhana, R., Lafreniere, D. et al. 2013, ApJ, 778, 36

Raghavan, D., McAlister, H.A., Henry, T.J. et al. 2010, ApJS, 190, 1

Ratzka, T., Kohler, R. \& Leinert, C. 2005, A\&A, 437, 611

Reipurth, B. \& Mikkola, S. 2015, AJ, 149, 145

Simon, M., Ghez, A.M., Leinert, C. et al. 1995, ApJ, 443, 625

Skemer, A., Hinz, P.M., Esposito, S. et al. 2012, ApJ, 753, 14

Spiegel, D. S., \& Burrows, A. 2012, ApJ, 745, 174

Stone, J., et al., 2016, ApJŁ, 818, 12

Thalmann, C., Desidera, S., Bonavita, M. et al. 2014, A\&A, 572, 91

Todorov, K., Luhman, K. L., \& McLeod, K. K. 2010, ApJ, 714, L84

van der Bliek, N.S., Manfroid, J., \& Bouchet, P. 1996, A\&AS, 119, 547

Winn, J.N. \& Fabrycky, D.C. 2015, ARAA, 53, 409 


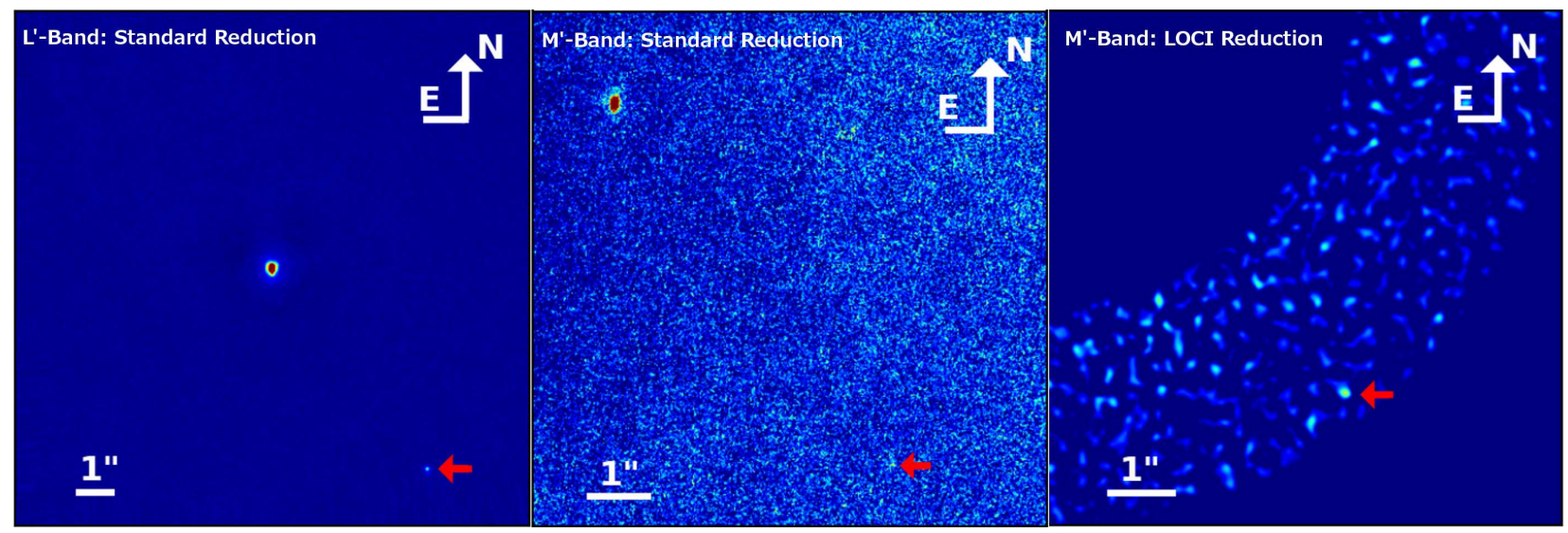

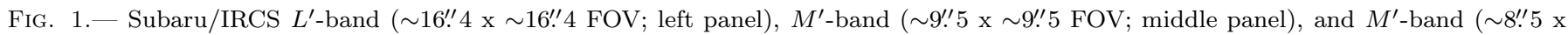
$\sim 8$ ". 4 FOV; LOCI reduction; 3 sigma filter applied; right panel) AO observations of the VHS 1256 system. Left and center panels show the full usable FOV with the partially resolved binary in the left and center panel and VHS 1256 b previously detected by Gauza et al. (2015) in all three panels. The red arrow depicts the location of VHS $1256 \mathrm{~b}$. Note that no other point sources are detected in the FOV. The data are plotted on a linear intensity scale.

$L^{\prime}$
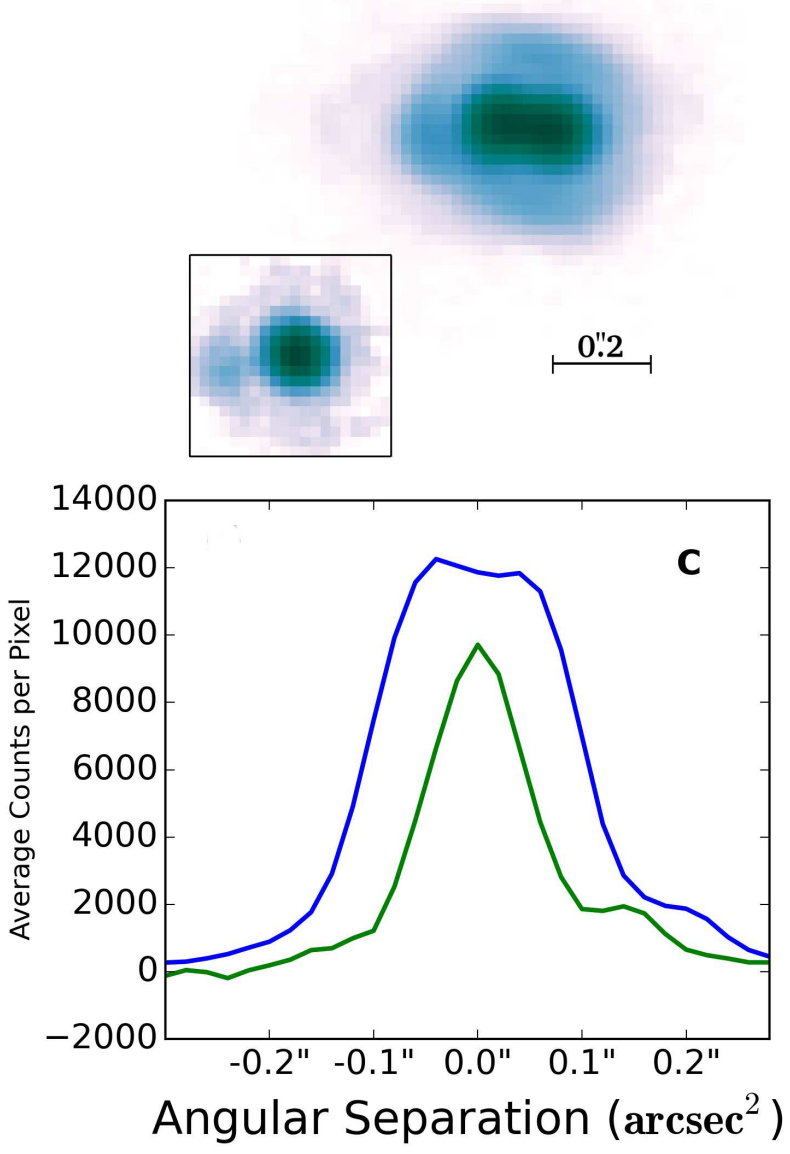

a

$\stackrel{0.2}{0.2}$
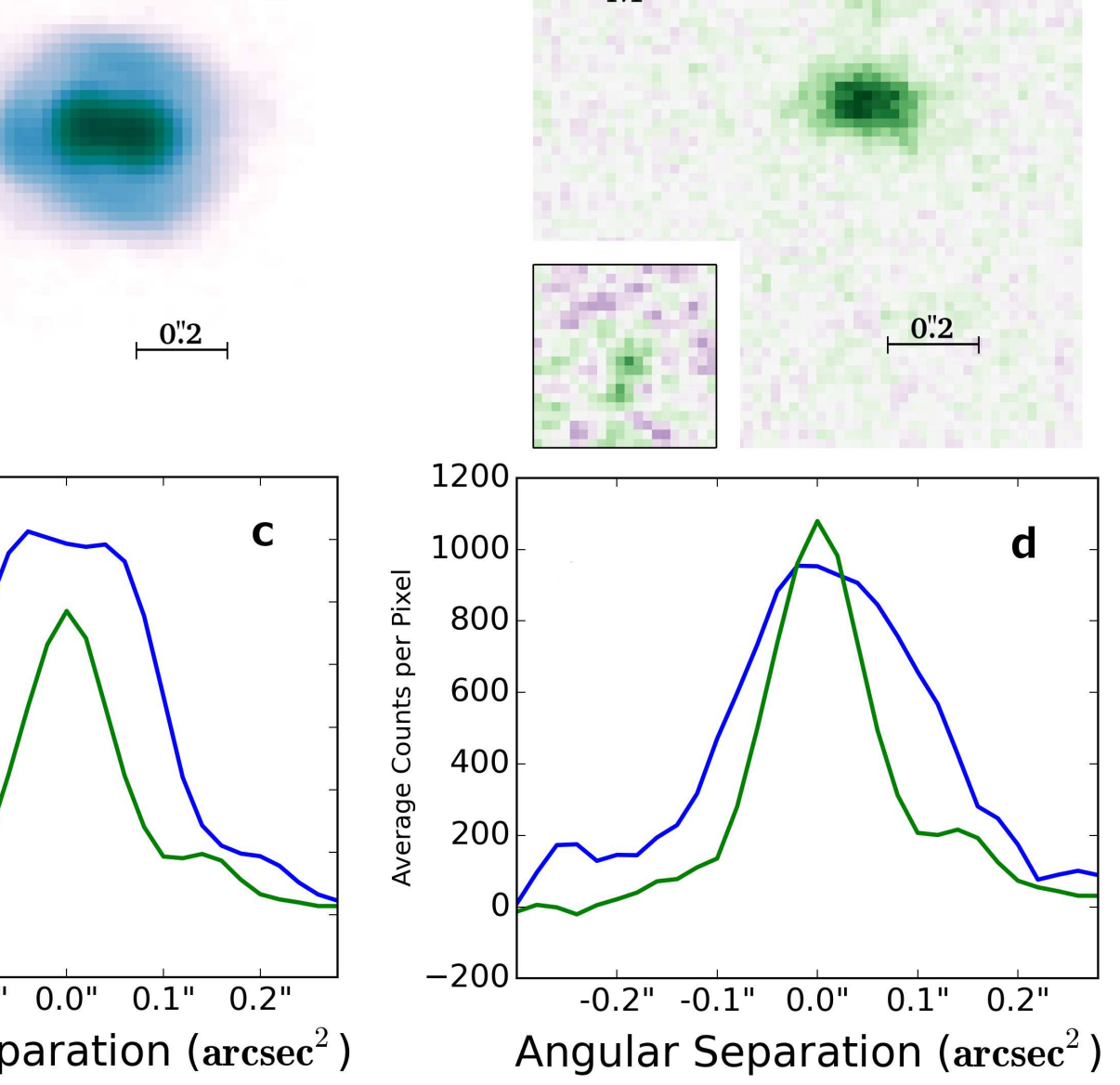

FIG. 2.- The ellipticity of VHS $1256 \mathrm{~A}$ and B in $L^{\prime}$ (panels a and c) and $M^{\prime}$ (panels b and d) can be seen above. Panels a and b show a FOV (1'. 3 X 1'! 3 ) of VHS $1256 \mathrm{~A}$ and B in their respective band-passes with north to the left and east up. The insert in panels a) and b) is of VHS $1256 \mathrm{~b}$ used as the PSF. Note that the panel b) insert used the A-LOCI reduction rather than the first reduction method. Panels c and $d$ are crosscuts along the major axis of the central source (blue) and PSF (green), averaged over 5 pixels. The clear double-peak profile in panel c illustrates that we partially resolve the central source in $L^{\prime}$. 


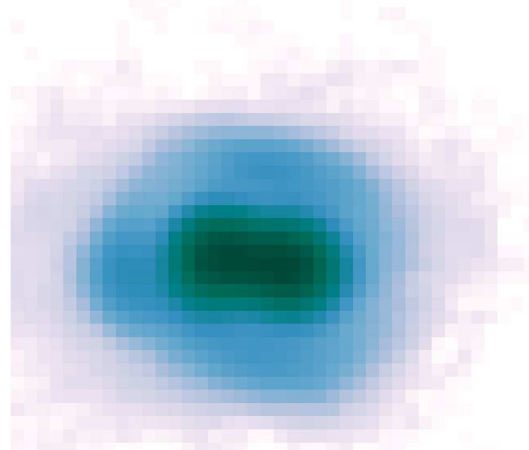

VHS $1256 \mathrm{~A}$ and $\mathrm{B}$

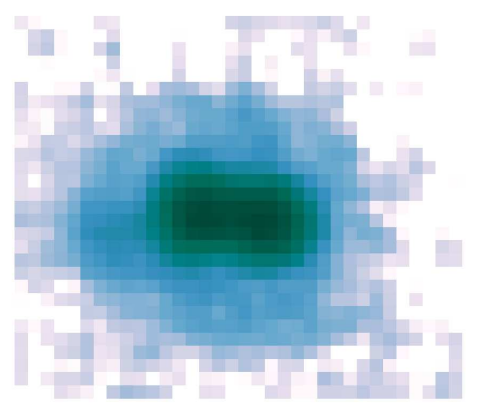

PSF fit

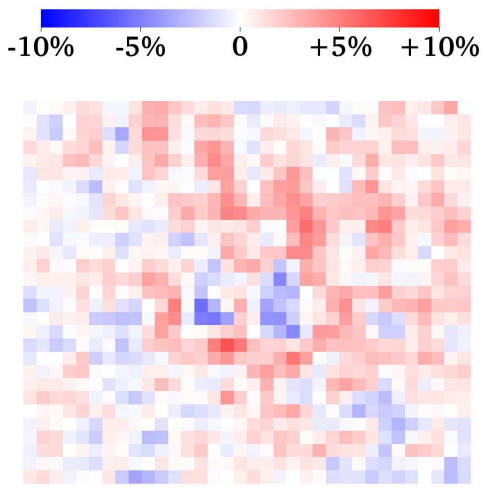

Residual

FIG. 3.- The above shows PSF fitting of VHS $1256 \mathrm{~A}$ and B utilizing our $L^{\prime}$-band data. The left panel shows a view of VHS $1256 \mathrm{~A}$ and B. The middle panel shows the best fit result using VHS $1256 \mathrm{~b}$ as an observed PSF. The right panel shows the residual of the PSF fit.
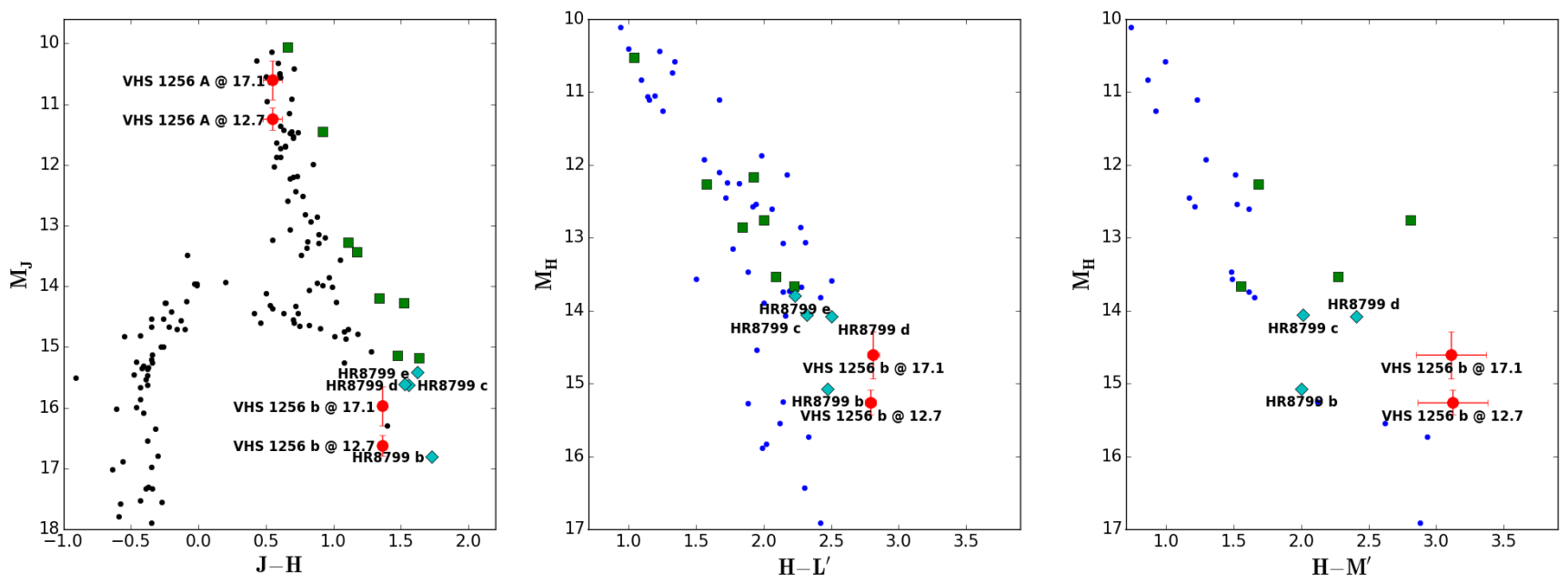

FIG. 4. - Near-IR color magnitude diagrams in J-H (right panel) and H- $M^{\prime}$ (left panel) space are shown. For both panels, the red circles are VHS $1256 \mathrm{~A}$ and b for both 12.7 and 17.1 pc distances ((Gauza et al. 2015; Stone et al. 2016)), the blue circles are HR 8799 bcde and L/T dwarfs with $M^{\prime}$ photometry (Leggett et al. 2002; Currie et al. 2014b), the green circles are members of the AB Dor moving group (Faherty et al. 2016), and the black cicles are M/L/T field dwarfs Dupuy \& Liu (2012). Note that VHS 1256 b is consistent in color with HR 8799 b in J-H space (right panel) but VHS $1256 \mathrm{~b}$ has a much redder color in $\mathrm{H}-M^{\prime}$ space (left panel).
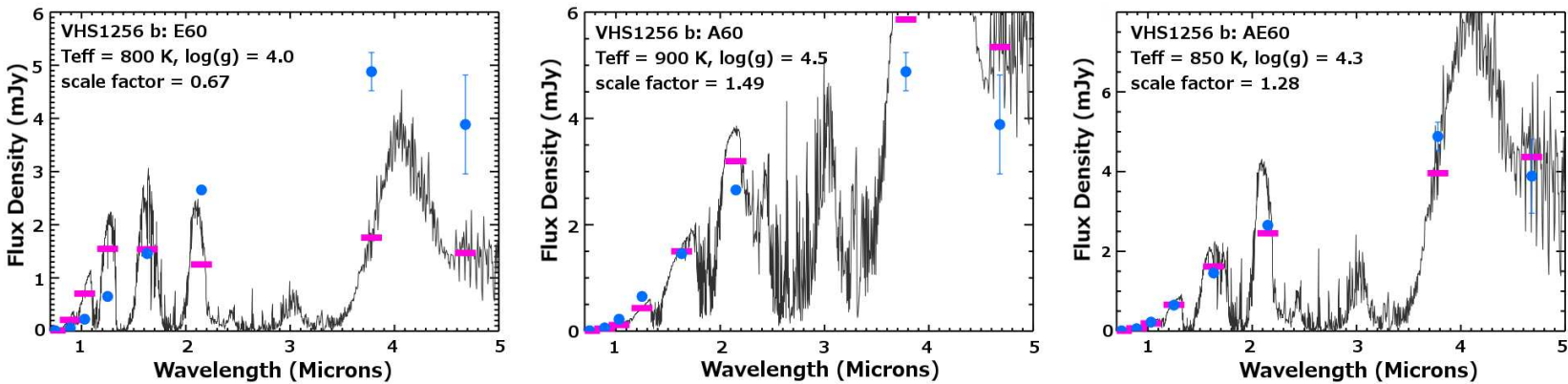

Fig. 5.- Best-fit atmosphere models for the thin-cloud E60 model (left panel), the very-thick cloud A60 model (middle panel), and the thick cloud AE60 model (right panel). The (very) thick cloud models accurately reproduce the optical to mid-IR SED of VHS1256 b. Black lines is the model spectra, the magenta points are the model spectra applied to the appropriate filter, and the blue points are photometry that match the magenta filter points. 

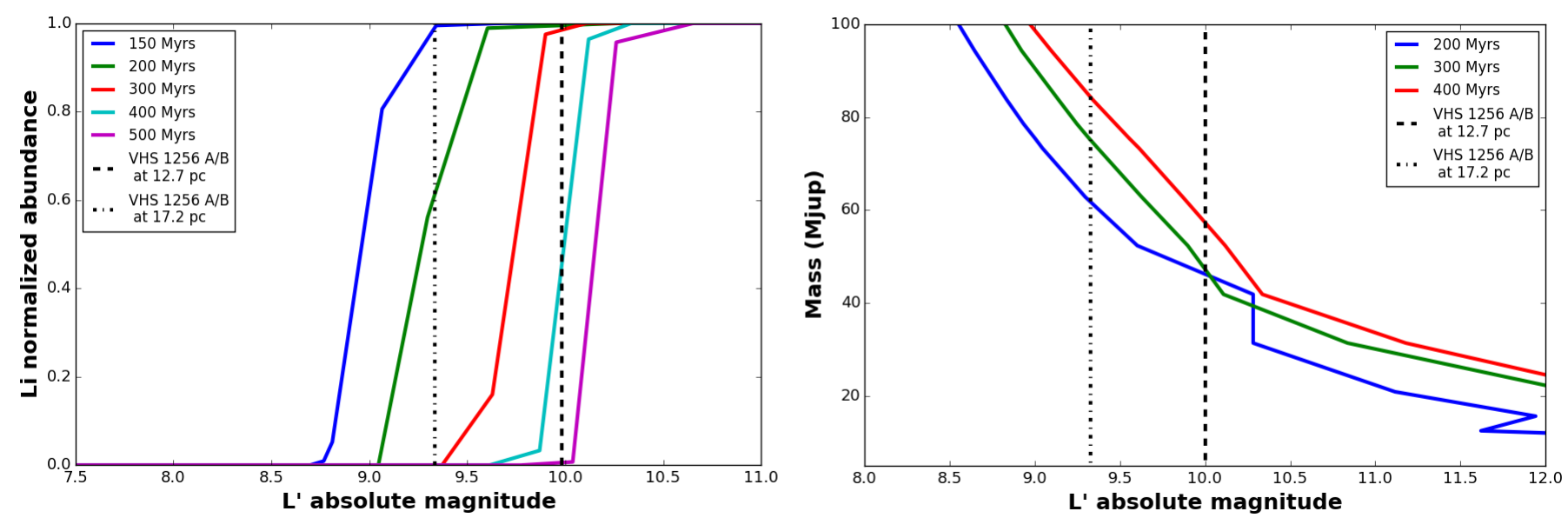

FIG. 6.- The above figures demonstrate the age of the VHS 1256 system (left panel), and the estimated lower mass limit of VHS 1256 A and B (right panel). The left panel plots the normalized lithium abundance against the absolute L' magnitude taken from BT-Settl models (Allard 2014) for a variety of ages. The absolute magnitudes of VHS $1256 \mathrm{~A}$ and B (both 12.7 and $17.1 p c$ distances) are taken from the PSF fit shown in table 5, plotted as the vertical dashed lines. Unresolved spectroscopy of stars A and B from Gauza et al. (2015) showed no detection of lithium down to $30 \mathrm{~m} \AA$, thus these stars must be old enough to have destroyed the initial lithium. The best lower limit ages based no the non-detection of lithium are $>200$ Myrs $(17.1 p c)$ or $>400$ Myrs $(12.7 p c)$. The right panel plots the sub-stellar mass $\left(M_{J}\right)$ against the absolute L' magnitude taken from BT-Settl models. The solid lines are three different BT-Settl models: 200 Myrs for the 17.1 pc distance, 400 Myrs for the $12.7 p c$ distance, and 300 Myrs for the Gauza et al. (2015) upper age limit. The estimated lower mass limit for VHD $1256 \mathrm{~A}$ and B for both 12.7 and $17.1 p c$ distances is $>58 M_{J}$.

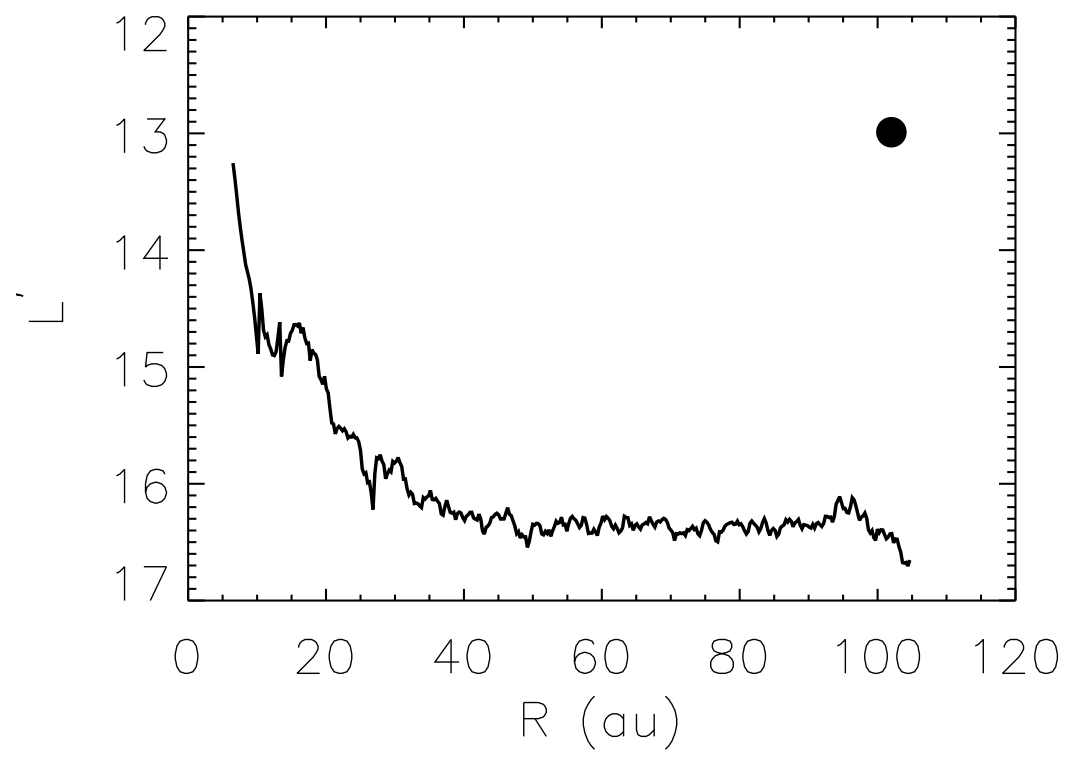

FIG. 7. - Limiting background limit as a function of projected radius from VHS $1256(\mathrm{~A}+\mathrm{B})$ for $L^{\prime}$ imagery with the A-loci reduction. The projected radius assumes a VHS 1256 distance of $12.7 \mathrm{pc}$ The black line is the 5-sigma background limit and the black dot is VHS $1256 \mathrm{~b}$. 
TABLE 1

VHS1256 Astrometry AND MaGnitudes

\begin{tabular}{cr}
\hline \hline VHS 1256 Objects and Band & System Properties \\
\hline A and B separation $\left(L^{\prime}\right)$ & $0^{\prime \prime} 1056 \pm 0.001$ \\
A and B PA $\left(L^{\prime}\right)$ & $-6^{\circ} \pm 2^{\circ}$ \\
A and B Est. Period $\left(L^{\prime}\right)$ & $4.7 \mathrm{yrs}$ \\
A apparent magnitude $\left(L^{\prime}\right)$ & $10.50 \pm 0.01 \mathrm{mag}$ \\
B apparent magnitude $\left(L^{\prime}\right)$ & $10.54 \pm 0.01 \mathrm{mag}$ \\
A+B and b separation $\left(L^{\prime}\right)$ & $8 . " 13 \pm 0 . .^{\prime} 04$ \\
A $+\mathrm{B}$ and b PA $\left(L^{\prime}\right)$ & $217.8 \pm 0.3^{\circ}$ \\
A+B and b separation $\left(M^{\prime}\right)$ & $8 . " 17 \pm 0.0^{\circ} 04$ \\
A $+\mathrm{B}$ and b PA $\left(M^{\prime}\right)$ & $217.8 \pm 0.3^{\circ}$ \\
\hline
\end{tabular}

Note. - The measured and derived for VHS $1256 \mathrm{~A}, \mathrm{~B}$, and $\mathrm{b}$ in $L^{\prime}$ and $M^{\prime}$ using centroid positions and PSF fitting.

TABLE 2

VHS1256 Aperture Photometry

\begin{tabular}{llc}
\hline \hline Photometry Band & $\begin{array}{l}\text { VHS 1256 A and B } \\
(\mathrm{mag})\end{array}$ & $\begin{array}{c}\text { VHS 1256 b } \\
(\mathrm{mag})\end{array}$ \\
\hline L' & $9.76 \pm 0.03$ & $12.99 \pm 0.04$ \\
M' & $9.65 \pm 0.05$ & $12.66 \pm 0.26$ \\
W1 & $9.880 \pm 0.023$ & $13.6 \pm 0.5$ \\
W2 & $9.658 \pm 0.021$ & $12.8 \pm 0.5$ \\
\hline
\end{tabular}

Note. - L' and M' aperture photometry taken from traditional reductions described in section 2 with the exception of M' photometry of VHS $1256 \mathrm{~b}$ which used the LOCI reduction described in section 2. The WISE data was taken from Gauza et al. (2015).

TABLE 3

Atmosphere Model Fitting Results (Freely-Varying Radius)

\begin{tabular}{llc}
\hline \hline \multirow{2}{*}{ Model } & Best-Fit Model & Models Matching Within 3- $\sigma$ \\
& $\mathrm{T}_{\text {eff }}(\mathrm{K}), \log (\mathrm{g})$, Radius $\left(R_{J}\right)$ & $\mathrm{T}_{\text {eff }}(\mathrm{K}), \log (\mathrm{g}), \operatorname{Radius}\left(R_{J}\right)$ \\
\hline Burrows/A60 & $1000,4.5,1.34$ & $1000,4.5,1.34$ \\
Burrows/AE60 & $800,3.8,1.8$ & $700,3.5-4,2.57-2.64$ \\
& & $750,3.5-4.3,2.03-2.25$ \\
& & $800,3.5-4.5,1.74-1.95$ \\
& & $850,3.5-4.3,1.60-1.64$ \\
Burrows/E60 & $1000,4.0,0.98$ & $900,3.5-3.9,1.35,1.42$ \\
\hline
\end{tabular}

Note. - Atmosphere modeling results shown in increments of $50 \mathrm{~K}$. The radii quoted assume the revised parallax from Stone et al. (2016). Owing to uncertainty in VHS 1256-12's parallax from that paper, the best-fit radii have an additional systematic uncertainty of $\approx 10 \%$. Radii assuming a nominal distance of $12.7 p c$ are systematically $\approx 35 \%$ smaller. 
TABLE 4

Atmosphere Model Fitting Results (Fixed Radius)

\begin{tabular}{|c|c|c|}
\hline Model & $\begin{array}{l}\text { Best-Fit Model } \\
\mathrm{T}_{e f f}(\mathrm{~K}), \log (\mathrm{g}), \text { Radius }\left(R_{J}\right)\end{array}$ & $\begin{array}{l}\text { Models Matching to Within } 3-\sigma \\
\mathrm{T}_{e f f}(\mathrm{~K}), \log (\mathrm{g}), \text { Radius }\left(R_{J}\right)\end{array}$ \\
\hline \multicolumn{3}{|l|}{$d=17.1 p c$} \\
\hline Burrows/A60 & $1000,4.25,1.45$ & - \\
\hline Burrows/AE60 & $850,3.8,1.55$ & $850,3.5-3.9,1.48-1.75$ \\
\hline Burrows/E60 & $700,4.0,1.45$ & - \\
\hline \multicolumn{3}{|l|}{$d=12.7 p c$} \\
\hline Burrows/A60 & $900,4.25,1.29$ & - \\
\hline \multirow[t]{4}{*}{ Burrows/AE60 } & $800,4.1,1.36$ & $750,3.5-3.8,1.53-1.73$ \\
\hline & & $775,3.8-4.1,1.35-1.53$ \\
\hline & & $800,4.0-4.3,1.25-1.41$ \\
\hline & & $850,4.3,1.26$ \\
\hline Burrows/E60 & $700,4.0,1.45$ & - \\
\hline
\end{tabular}

Note. - Atmosphere modeling results, shown in increments of $25 K$. As before, for a given parallax assumption the best-fit radii have an additional systematic uncertainty of $\approx 10 \%$.

TABLE 5

VHS1256 Absolute Magnitudes And Masses

\begin{tabular}{|c|c|c|}
\hline Object & $\begin{array}{l}\text { Measurement } \\
\text { at } 12.7 \pm 1.0 \mathrm{pc}\end{array}$ & $\begin{array}{l}\text { Measurement } \\
\text { at } 17.1 \pm 2.5 \mathrm{pc}\end{array}$ \\
\hline A Absolute magnitude $\left(L^{\prime}\right)$ & $10.0 \pm 0.2 \mathrm{mag}$ & $9.3 \pm 0.3 \mathrm{mag}$ \\
\hline B Absolute magnitude $\left(L^{\prime}\right)$ & $10.0 \pm 0.2 \mathrm{mag}$ & $9.4 \pm 0.3 \mathrm{mag}$ \\
\hline Age lower limit & $>400$ Myrs & $>200$ Myrs \\
\hline A and B Mass BT-Settll ${ }^{a}$ (L') & $58 \mathrm{M}_{J}$ & $58 \mathrm{M}_{J}$ \\
\hline b Absolute Magnitude (L') & $12.5 \pm 0.2 \mathrm{mag}$ & $11.8 \pm 0.3 \mathrm{mag}$ \\
\hline b Mass BT-Settl ${ }^{a}\left(\mathrm{~L}^{\prime}\right)$ & 26.2 to $74 \mathrm{M}_{J}$ & 15.7 to $75 \mathrm{M}_{J}$ \\
\hline b Bolometric Luminosity $\log \left(\mathrm{L} / \mathrm{L}_{\odot}\right)$ & -5.06 to -5.24 & -4.79 to -4.95 \\
\hline b Mass BT-Settl ${ }^{a}$ Bolometric & $12.6-20.6 \mathrm{M}_{J}$ & $10.5-15.7 \mathrm{M}_{J}$ \\
\hline Limiting Absolute Magnitude at 2.! 8 (L') & 15.6 & 14.9 \\
\hline Limiting Magnitude Mass SB12 ${ }^{b}$ models (L') & $\sim 10 \mathrm{M}_{J}$ & $\sim 10 \mathrm{M}_{J}$ \\
\hline Limiting Magnitude Mass BT-Settl ${ }^{a}$ models (L') & $5 \mathrm{M}_{J}$ & $7.8 \mathrm{M}_{J}$ \\
\hline
\end{tabular}

Note. - The measured and derived parameters for VHS $1256 \mathrm{~A}, \mathrm{~B}$, and b in $L^{\prime}$ and $M^{\prime}$. a) BT-Settle model from Allard (2014). b) SB12 models from Spiegel \& Burrows (2012). 